\title{
The Application and Design of Big Data in Operation and Maintenance of Industry 4.0
}

\author{
Jiqing $\mathrm{CaO}^{1, \text { a }}$, Shuhai Zhang ${ }^{2, \mathrm{~b}}$ \\ ${ }^{1}$ Department of Information Engineering, Suzhou Industrial Park Institute of Service Outsourcing, \\ Suzhou, 215123, China \\ ${ }^{2}$ Department of IT Management, Bosch Automotive Products (Suzhou) Co., Ltd., Suzhou, 215124, \\ China \\ aemail: George.cao@siso.edu.cn, bemail: Zhangsh@163.com
}

\begin{abstract}
Keywords: Industry 4.0; Operation and Maintenance; Big Data; Architecture Design; Cloud Computing
\end{abstract}

\begin{abstract}
Industry 4.0 system generates vast amounts of data in the Operation and Maintenance process. To explore the value of these data is the key to achieve the goals and values of Industry 4.0. This paper discusses the various application scenarios of Big Data for the Operation and Maintenance process of Industry 4.0, including the predictive maintenance of failures, production optimization, product innovation, supply chain optimization, performance monitoring, quality management and secure handling of information, and other aspects. To achieve the formation of industrial Big Data and its application, the paper designs three-tier architecture of the Big Data management platform including data acquisition, storage, analysis, processing and application service providing which integrates data from disparate systems. Through the effective analysis of these industrial data on the platform, it can achieve the relative business services provided to the users of the Industry 4.0 system. The architecture of the Big Data platform has guided the practice of the Operation and Maintenance in the cooperative enterprises and has significantly increased the efficiency of their Operation and Maintenance works.
\end{abstract}

\section{Introduction}

ITIL (Information Technology Infrastructure Library) is the fact international standard of Operation and Maintenance. In ITIL, Operation and Maintenance is called Service Operation and defined as a stage of IT Service Management Lifecycle, and is responsible for ensuring the normal operation of the business activity. The ubiquitous sensing and connecting during production operations of Industrial 4.0 bring massive data. Having massive data is not the goal, to explore its value is the key. Aggregated from enterprise information data, things data and the external Internet industrial data, the industrial data contain tremendous value.

Industry 4.0 has three salient features, they are (1) Agile and Lean production. It not only needs to ensure the product quality, but also achieve the flexible production of the co-ordination among the design, manufacture and the user requirements; (2) Self-aware Capability of the system. The system can be automatically adjusted according to the changed processing status in the manufacturing process; (3) Failure Prediction and Auto Repair. It needs to achieve zero faults, zero risk and zero accidents throughout the manufacturing process [1]. These requirements for Industry 4.0 value as automation, intelligence, real-time, as well as multi-objective optimization method of controlling all need to use the Big Data technology.

This paper is an attempt to explore the Big Data applications in Operation and Maintenance process of Industry 4.0, to design a unified platform for Big Data acquisition, analysis and service providing, and to promote the efficiency, intelligence, security monitoring and decision-making of the Operation and Maintenance in Industry 4.0. 


\section{Big Data Applications in Operation \& Maintenance of Industry 4.0}

Industrial data applications will bring industrial innovation and a new era to the enterprises and create more value for them. Typical applications for industrial data in the Operation and Maintenance of Industry 4.0 include Failure Predictive Maintenance, Production Optimization, Product Innovation, Supply Chain Optimization, Operation Monitoring, Quality Management and Information Security Processing.

(1) Failure Predictive Maintenance

Industry 4.0 systems must be able to independently assess the state of health, and to make maintenance decisions in order to avoid potential system failures. With technologies of Big Data, cloud computing and automation, it can avoid unplanned outages through condition-based maintenance, achieve self-management through the production equipment exchange between devices, monitor wear, degradation and other fault conditions, and immediately take corrective actions. [2]

Using Big Data technology, IBM and American National Instruments Company have developed a related technology to continuously and successfully monitor the early signs of degradation or failure of the devices, so as to determine the cause and repair range of the potential failure of the device and achieve the value and benefit of the industrial equipment. [3]

In addition, Big Data also give us the new means of fault analysis. Through the Correlation Analysis between the equipment maintenance history data and its use conditions with Big Data technology, it can help find the real reason of the equipment failure, and continue to improve the health of the equipment and the lean management. [4]

(2) Production Optimization

The Self-aware function of the system is one of the important characteristics of Industry 4.0. System self-aware means the system can automatically adjust during the manufacturing process according to the status changes. To realize the adaptive control of the system, we need to use the Big Data to analyze the data of the equipment and its surrounding environment, process in real-time and feedback to the device controller. The information is also fed back to the facility engineers to facilitate timely maintenance. [5]

Flexible production mode with more variety, small batch and fast response is a huge challenge to the Industry 4.0 production. Big Data can find deviations between the forecast and the actual state, balance and adjust the workload and work pressure of each device according to the health condition of each device, develop the pre-production plan and schedule by the intelligent optimization algorithm, and adjust the production plan dynamically and optimally. [6]

(3) Product Innovation

The interaction between customers and industrial enterprises will generate large amounts of data which can help the customers to participate in product demand analysis and product design innovation activities through mining and analyzing these dynamic customer data and contribute to product innovation.

Ford Motor Company has applied Big Data technology to the optimization and innovation of their new electric automobile. They collect and analyze large amounts of data in the use of electric vehicles, such as information of acceleration, braking, battery charge, and location, with which they develop the product improvement plan, and implement continuous innovation of new products. [7]

(4) Supply Chain Optimization

RFID (Radio Frequency Identification), Internet technology as well as mobile Internet technology can help industrial enterprises to obtain a complete Big Data of the product supply chain. It can enhance the forecasting accuracy of the demand of the spare parts and significantly improve the efficiency of the storage, distribution and marketing, and significantly decrease costs after analyzing the use of these data. Based on the data generated by the sensors of equipment and products, they can know the risk and the location of the failures, so that they can also predict where and when they need spare parts, which will greatly reduce inventory and optimize the supply chain.

In all aspects of Haier supply chain, customer data, internal data, supplier data are aggregated to the supply chain system, the company can continuously improve and optimize the supply chain to 
ensure the agile and quick respond to the customer through the Big Data analysis of the supply chain. [8]

(5) Real-time Operation Monitoring

The Big Data technology can collect the location, condition and event information of the equipment and the system in real-time. With visualization techniques, the Operation and Maintenance staff will be able to timely grasp the current operating status of the system without being onsite. With these monitoring data, it can achieve a variety of analysis, including production status, system performance, energy analysis, quality accident analysis (including violation of the production provisions, parts failure) and etc.

First, it will be able to analyze the entire production process with these Big Data for the improvements in the production process. When there is a deviation from the standard process flow, it will generate an alarm signal so that it can more quickly find errors or bottlenecks and make it easier to solve the problem. Secondly, the Big Data technologies can also create a virtual model to simulate and optimize the production processes. When all processes and performance data can be rebuilt in the system, the transparency will help companies improve their production process. Furthermore, in the analysis of energy consumption, it can find unusual circumstances of energy consumption with the sensors centralized monitoring of all production processes, so as to optimize the energy consumption of the production process. It will significantly reduce energy consumption when all processes are analyzed. [9]

(6) Product Quality Management

Highly automated equipment will generate huge production and test data in the course of processing products. Big Data technology can analyze these vast amounts of data in order to accurately identify the key reasons for fluctuations in product quality. According to the basic methods of traditional quality management, it will cost huge workload to do the process capability analysis for these massive data, it is still difficult to see the correlation from such a large number of process capability indexes, and harder to have an integrated understanding for the overall product quality performance. However, Big Data can easily implement these analyses. [10]

(7) Information Security Management

In the era of Industry 4.0, IT system security involves not only the operational aspects of the production, but also linked to the communication network extending therefrom. If it can not secure the production system of the manufacturing enterprise, user privacy and data security, Big Data would be nothing.

In the process of Operation and Maintenance of Industry 4.0, it can establish monitoring and early warning measures of an effective security event with the Big Data technologies of Threat Discovery, Authentication, Data Authenticity Analysis, and Security as a Service. It can quickly and accurately find aggressive behavior, and quickly start the response mechanisms when the information systems are about to be attacked or has been under attacked. Through the security incidents and comprehensive analysis of the security situation with Big Data technologies, it can draw the rules of relationship between the macro law and the different types of risk events, and provide effective data support and decision support for the whole network and information security planning. [11]

\section{Architecture Design of Operation and Maintenance Big Data Platform}

For manufacturing enterprises, strategic significance of Big Data technology lies not only in having huge data, but also the transformed valuable information after the specialized treatment on these data. To build a unified Big Data platform in an industrial environment can help the plants improve the ability of data mining, collect vast amounts of information from different devices, improve the computing power and the ability to digest the data of the enterprise information system, and achieve the real-time analysis of the enterprise product data, operation data, sales data and customer data. It can be used to guide the next round of research and development, production, sales and services, which will enable companies to implement the on-demand production and improve their operation efficiency. 
To implement the application of the industrial Big Data, this paper designed the logic architecture (see Figure 1) of the integrated Big Data Platform for the Operation and Maintenance. It consists of three layers, namely Service Provider Layer, Analysis and Processing Layer, Acquisition and Storage Layer, covering data collection, transmission, storage, analysis, processing and services based on the cloud computing platform. All the data from production, logistics, quality, and Operation and Maintenance will be integrated on the platform by the distributed cloud storage technology. All types of the data will be managed effectively so as to achieve predictive failure and repair, manufacturing and supply chain optimization, real-time Operation and Maintenance monitoring and improving the processing capacity requirements of information security.

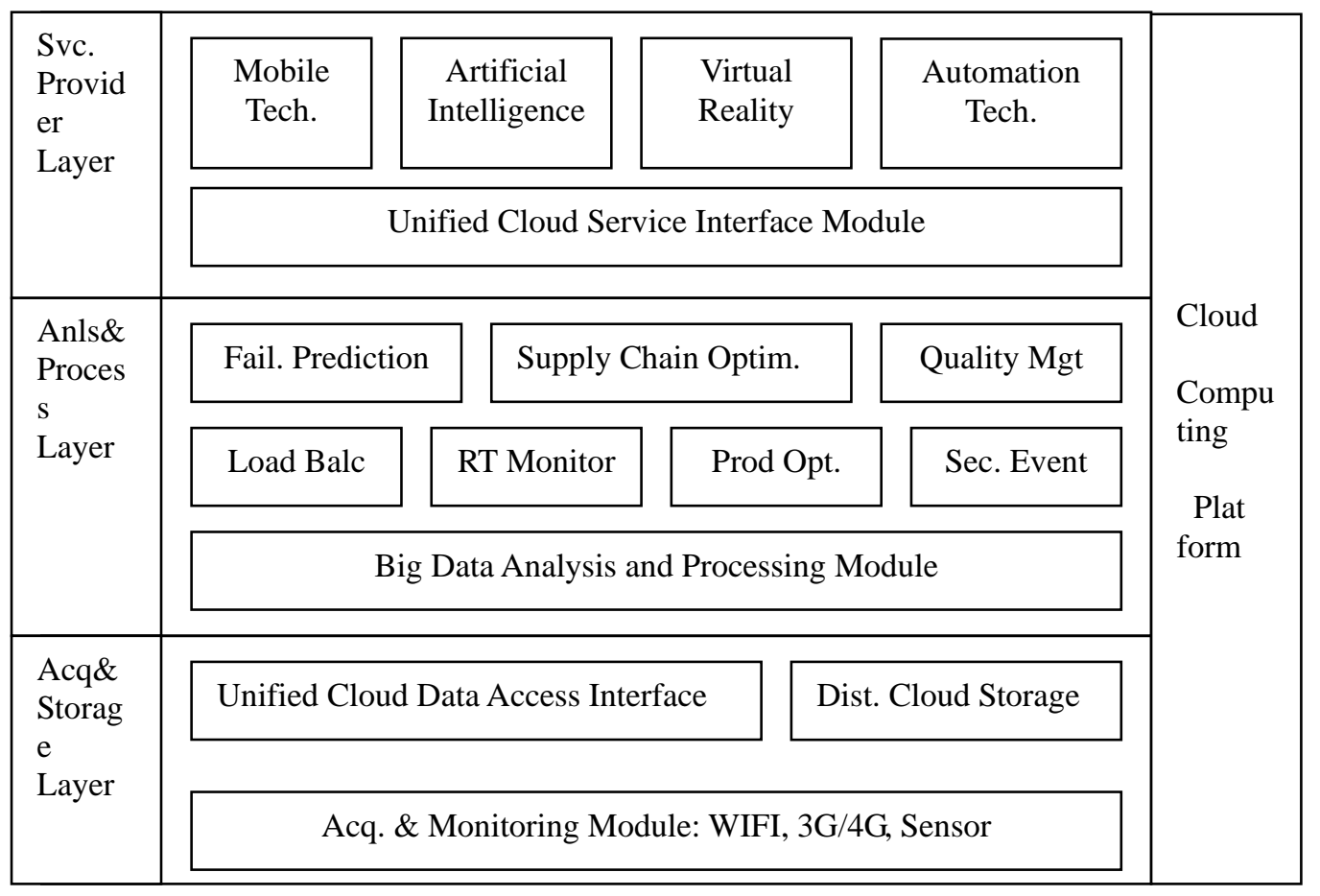

Fig.1. Logic Architecture of Big Data Platform for Operation and Maintenance

(1) Acquisition and Storage Layer

Based on the distributed cloud storage technology, the Big Data from the production system, management system and Internet are collected through various networks and sensors, and realize the distributed storage and management of the multi-source, heterogeneous and large amounts of data. This layer will provide a Unified Cloud Data Access Interface to the Analysis and Processing Layer.

The architecture based on the cloud storage can solve the storage problem of the Big Data. Cloud storage network can integrate the large number of the storage devices with different types and make them work together. Cloud storage is storage of full virtualization, all devices on the cloud are completely transparent to the users, any authorized systems or users can connect to the cloud storage via a network and store data in the entire piece of the cloud. It breaks the bottlenecks of the traditional storage and achieves a linear expansion of performance and capacity.

(2) Analysis and Processing Layer

Using suitable Big Data analysis techniques and tools, the layer analyzes and processes the production information, quality information, energy consumption and cost data etc., in order to achieve data insight and a variety of applications. Common applications include Fault Predictive Maintenance, Production Optimization, Product Innovation, Supply Chain Optimization, Real-time Performance Monitoring, Quality Management and Information Security Processing, which open the call to the Service Layer.

Data mining on Big Data required the entire process to extend to both the front and the rear according to the Big Data characteristics, including the specific process for processing and analysis from a massive, multi-source Big Data, patterns, rules and knowledge for auto-discovery and 
extraction of implicit, and present through visual and easy-to-understand manner. Data mining has a relatively large degree of difficulty. It needs the support of theories of the intelligent reasoning and other related technologies based on Big Data and knowledge base.

(3) Service Provider Layer

The Layer provides a unified cloud service interface to Industry 4.0 systems and users with various types of Big Data application services. It displays the systems conditions in real-time with the visual technologies like Virtual Reality, and achieves the real-time alarming of the failures. It also achieves remote automated repair of the risks and failures, and the optimization solution of the production and supply chain with the automation, artificial intelligence and cloud computing technologies, etc.

\section{Practice of the Big Data Platform Architecture}

As a pioneer of Industry 4.0, SAISI Automotive Products (Suzhou) Company is a representative of auto parts manufacturers. The company makes the CNC equipment, robots, auxiliary materials and equipment of its flexible production line intelligent and linked, each production line is deployed a production line console subsystem, and the background systems share the server of the production line. The information of manufacturing process, material staff and production data of the company are saved in the server side.

In accordance with the architecture of the designed Big Data platform, SAISI built its distributed Big Data management platform for the Operation and Maintenance of its Industry 4.0 system. Based on WIFI and 4G wireless network, it achieved real-time collecting and monitoring of the intelligent devices and the Big Data of the production status. Based on the cloud storage, it achieved the storage of the massive production data. Based on the Big Data and relative technologies, it achieved the real-time analysis on these large data. Based on the unified interface of the cloud service platform, Virtual Reality and Artificial Intelligence technologies, it achieved the real-time alarming and automatic repair for risks and failures, the automatic operation of the multiple manufacturing processes when the different parts change, the real-time monitoring of the production process and production line optimization, and the intelligent controlling of the flexible production line when facing the single or mass production.

\section{Summary}

Big Data is the key to explore the value of the huge amounts of the data of Operation and Maintenance process and the thinking hub of the entire Industry 4.0 system. This paper lists a variety of application scenarios and success cases of the Big Data in the Operation and Maintenance process of Industry 4.0, but the majority of these cases have not formed an integrated whole application, for example, only the individual application in failure prediction or supply chain optimization, etc. Therefore, this paper designed logic architecture of the Big Data Management Platform including the Big Data acquisition, storage, analysis, processing and services provider, which is able to guide enterprises to integrate data from different systems, be focused on data analysis and processing these industrial Big Data, and provide the appropriate services for Industry 4.0 systems through a unified interface of the cloud services. The architecture has well guided the practice of the Operation and Maintenance works in the virtualization environment for the cooperative enterprises, proved to be feasible and reliable, but it should be noted that, in order to fully realize the value of the Big Data, many problems needs to be further solved such as Big Data mining technology, data quality, infrastructure and talents.

\section{Acknowledgement}

In this paper, the research was sponsored by the Service Outsourcing Foundation of Suzhou Industrial Park Institute of Service Outsourcing (Project No. KY-XJY03). 


\section{References}

[1] LI Jie. Industry 4.0 Age: China's Opportunity Lies in Data and Data Analysis [J]. World Science, 2014(6)

[2] LI Jie. Cheng Qisen. Predictive Manufacturing under Industry 4.0 Environment [J].World Science, 2014(5)

[3] NIWeek 2015. Big Data Open the Door of Internet of Things [J].Computer Measurement \& Control, 2015(9)

[4] HE Zhenwei.Yu Lei.Zhou Shouqin.Wang Jiqing.Pu Hanjun. the Whole Life-cycle Management System Development of the Compressor Based on the Industrial Big Data[J]- Automation \& Information Engineering, 2014(6)

[5] Jay LEE.Behrad BAGHERI. Trends of Big Data Analytics and Cyber-Physical Systems in Industrial 4.0 Systems, The 5th International Conference on Mechanical Engineering and Mechanics, 2014

[6] HUANG Jun.CUI Yamei. Project Management Used in Custom-manufacturing Enterprises- in ZJFC's Case [J]. China Collective Economy, 2016(1)

[7] LI Zhongdong. Ford's Move to Create a New Model Car [J]. Automobile \& Parts, 2015(15)

[8] HE Jiao. Big Data Applications in JIT Procurement [J]. Logistics Engineering and Management, 2014(7)

[9] FAN Fulin. Optimization of Industry under Big Data Platform [J]. China Plant Engineering, 2015(6)

[10] ZHANG Weidong.MA Chuang Tao. the Forecasting Method of Pinning Quality Abnormal Factor Based on the Real-time Data [J], Advanced Textile Technology, 2015(5)

[11] FENG Dengguo.ZHANG Min.LI Hao. Big Data Security and Privacy Protection [J].Chinese Journal of Computers, 2014(1)

[12] MO Ying.DUAN Yongli.LIANG Xinnuo.YUAN Chunmei. Industry 4.0 and Big Data Applications: a War without Gunpowder [J]. Textile Machinery, 2015(10) 